\title{
Clostridial myonecrosis in association with etoposide therapy presenting as severe thigh pain
}

\author{
D. Smith ${ }^{1}$, M. Harris ${ }^{2}$ and N. Thatcher ${ }^{1}$ \\ ${ }^{1}$ Cancer Research Campaign Department of Medical Oncology, and ${ }^{2}$ Department of Pathology, Christie Hospital \\ and Holt Radium Institute, Manchester M20 9BX, UK.
}

\begin{abstract}
Summary: The case is described of a 68 year old man who was immunosuppressed as a result of receiving chemotherapy for small cell lung cancer and who presented with severe right thigh pain. This proved to be due to clostridial myonecrosis with none of the classical clinical features of clostridial infection. This is believed to be the first reported case of spontaneous clostridial infection associated with etoposide administration.
\end{abstract}

\section{Introduction}

Patients with malignant disease often present with pain for which there may be many explanations. We describe the case of a man receiving chemotherapy for small cell (oat cell) lung carcinoma who presented with severe pain in the right thigh which subsequently proved to be due to clostridial myonecrosis.

\section{Case report}

Following a bronchoscopic diagnosis of small cell lung carcinoma, a man, aged 68 , was entered into the current treatment protocol for this tumour, involving three courses of cyclophosphamide $\left(2.5 \mathrm{~g} / \mathrm{m}^{2}\right.$ i.v. day 1) and etoposide $\left(120 \mathrm{mg} / \mathrm{m}^{2}\right.$ i.v. day $1,240 \mathrm{mg} / \mathrm{m}^{2}$ orally days 2 and 3 ) repeated at 21 day intervals (Thatcher et al., 1985). Pretreatment haematology and biochemical profile were normal but his bone marrow (aspirate and trephine from the left iliac crest) was involved with tumour. He received cyclophosphamide $4.8 \mathrm{~g}$ and etoposide $200 \mathrm{mg}$ on day 1 with etoposide $400 \mathrm{mg}$ on days 2 and 3 . Two weeks later he was admitted with a 72 hour history of constant severe pain in his right hip and thigh. Physical examination was normal. He was apyrexial, blood pressure 130/ $80 \mathrm{~mm} \mathrm{Hg}$, pulse rate $90 / \mathrm{min}$ and routine urinalysis was normal. His white cell count was $1.2 \times 10^{9} / 1(26 \%$ neutrophils), haemoglobin $12.4 \mathrm{~g} / \mathrm{dl}$ and platelets $239 \times 10^{9} / 1$, but the serum alkaline phosphatase was now $359 \mathrm{IU} / 1$ (normal range 25-110 IU/1). The pain was considered to be due to bone metastases and he was treated with analgesics but 13 hours later he died

Correspondence: D. Smith, M.R.C.P.

Accepted: 22 October 1985 peacefully in his sleep. Immediately after death external examination of the body was normal.

An autopsy was conducted 8 hours after death, the body having been refrigerated in the interim. The right upper thigh had become swollen and tense and the abdomen and scrotum were distended due to the presence of gas under tension. The right psoas and quadriceps muscles were swollen, soft and friable. Small blood vessels throughout the body contained gas bubbles and the liver contained vast numbers of tiny gas cysts. In the intestinal tract there were old fibrous adhesions between a loop of ileum and the posterior parietal peritoneum on the right side but there was no lesion to account for this unusual finding. The only detectable tumour was a necrotic mass at the hilum of the left lung. Histological sections of all organs examined showed many gas cysts with a multitude of large Gram positive bacilli in blood vessels and interstitial tissues. The quadriceps muscle showed patchy necrosis but very little inflammatory cell infiltration. The lung tumour was extensively necrotic with only a few recognisable tumour cells. Cultures of spleen, liver and quadriceps muscle grew Clostridium perfringens and Escherichia coli. The muscle also grew $C$. paraputrificum.

\section{Discussion}

Non-traumatic clostridial infections, though rare, are more likely to occur in patients with malignant disease who are frequently immunosuppressed and may have disordered tissue metabolism (Scully et al., 1979; Wynne \& Armstrong, 1972). The organism may produce myonecrosis at a site distant from any portal 
of infection, occur as a visceral anaerobic cellulitis or as a primary intra-abdominal infection extending along the ileo-psoas muscles to the buttock and thigh (Scully et al., 1979). Our patient appears to fall into the latter group.

Clostridium perfringens is the commonest organism isolated (Scully et al., 1979) occurring alone in cases of distant myonecrosis, but in combination with aerobic species in patients with primary intra-abdominal infections, thus reflecting the bowel flora from which they are derived. The route of infection in these cases is thought to be through breaks in the bowel mucosa as a result of the action of cytotoxic drugs (Jendrzejewski et al., 1978). Etoposide is particularly associated with mucosal ulceration (Achterrach et al., 1982) in con-

\section{References}

ACHTERRACH, W., NIEDERLE, N. \& HILGARD, P. (1982). Etoposide - chemistry, preclinical and clinical pharmacology. Cancer Treatment Reviews, 9 Suppl. A, 3.

JENDRZEJEWSKI, J.W., JONES, S.R., NEWCOMBE, R.L. \& GILBERT, D.N. (1975). Non-traumatic clostridial myonecrosis. American Journal of Medicine, 65, 542.

SCULLY, E.R., GALDABINI, J.J. \& MCNEELY, B.U. (1979). Case Records of the Massachusetts General Hospital. New England Journal of Medicine, 301, 1276. trast to cyclophosphamide which rarely causes this complication. Moreover there has been an unusually high incidence of perianal sepsis in patients treated with etoposide for small cell lung cancer in this hospital (Thatcher et al., 1985). This suggests that etoposide might be associated with clostridial infection; however, no previously reported cases could be found.

In this case the classical symptoms and signs of gas gangrene (Jendrzejewski et al., 1978) were absent emphasizing the need to consider such a diagnosis in immunocompromised patients, particularly if pain is disproportionate to accompanying clinical features. Broad spectrum antibiotics should be commenced on the earliest suspicion.

THATCHER, N., STOUT, R., SMITH, D.B., ,GROTTE, G. WINSON, M., BASSETT, H. \& CARROLL, K.B. (1985). Three months treatment with chemotherapy and radiotherapy for small cell lung cancer. British Journal of Cancer, 52 , 327.

WYNNE, J.W. \& ARMSTRONG, D. (1972). Clostridial septicaemia. Cancer, 29, 215. 